\title{
A Case of Empyema and a Review of Practice in a District General Hospital
}

\author{
Authors: \\ *Avinash Aujayeb, Kevin Conroy \\ Northumbria Healthcare NHS Foundation Trust, UK \\ *Correspondence to Avinash.aujayeb@nhct.nhs.uk \\ Disclosure: $\quad$ The authors have declared no conflicts of interest. \\ Received: $\quad 31.03 .19$ \\ Accepted: $\quad 13.05 .19$ \\ Keywords: $\quad$ Empyema, pleural infection, pleural fluid. \\ Citation: $\quad$ EMJ Respir. 2019;7[1]:91-96.
}

\begin{abstract}
The authors describe an empyema in an immunosuppressed patient. Thoracentesis was attempted and only $60 \mathrm{~mL}$ of pus was obtained from her pleural space. She was treated as an outpatient with antibiotic therapy. The authors have recently performed a review of all cases of pleural infection between December 2016 and December 2017 in their trust, of which there were 36. Here, they describe failings that have now been addressed and which helped in managing this particular case. As a result of this review, the authors have developed a pleural procedure form that encompasses all of the recommendations from their case review.
\end{abstract}

\section{BACKGROUND}

The Northumbria Healthcare NHS Foundation Trust serves just over half a million people across four different sites. There is a well-established pleural service. ${ }^{1}$ Approximately $57 \%$ of patients with pneumonia develop a pleural effusion, which normally resolves with antibiotics. Due to a number of factors, including bacterial translocation and activation of inflammation via cytokine production, fibrin strands and locules can develop. Empyema, i.e., pus in the pleural space, can result and is a progressive process from a simple exudate to fibrinopurulent stage, before commencing towards an organising stage with pleural peel formation. ${ }^{2}$ Clinical outcomes remain poor, with up to $20 \%$ requiring surgery and up to $30 \%$ of frail, elderly, or immune-compromised patients dying in the first year. Length of stay in hospital varies between 12 and 21 days, and the overall incidence is about 1.98 per 1,000 in the UK. ${ }^{2}$

\section{CASE PRESENTATION}

A 75-year-old female patient presented to her general practitioner with a 2-week history of cough, fevers, decreased appetite, and purulent green phlegm. Her past medical history included psoriatic arthritis controlled by azathioprine and hydroxychloroquine; Type 2 diabetes mellitus on insulin, metformin, and gliclazide; and hypertension controlled by atenolol, irbesartan, and furosemide. She had never been a smoker. She had a temperature of $39.7{ }^{\circ} \mathrm{C}$ but was normotensive. Heart rate and oxygen saturations on room air were normal. She was referred to the medical ambulatory care department.

\section{INVESTIGATIONS}

A chest radiograph showed a large right sided pleural effusion (Figure 1A). A CT showed typical features of empyema with pleural enhancement, a 
lenticular effusion, and internal loculations (Figure 2A). A thoracic ultrasound showed a hyper-echoic effusion with significant loculations (Figure 2B). Thoracentesis under aspesis was attempted. Only $60 \mathrm{~mL}$ of frank pus was aspirated. Two further attempts in different intercostal spaces were unsuccessful.

The pus was sent for analysis in both a white topped bottle and a blood culture bottle. A repeat chest radiograph post aspiration showed a smaller effusion with a central air containing space. The patient's oxygen saturations remained normal. Her white cell count was $12.4 \times 10^{9}$ cells $/ L$ (4.0-11.0), urea concentration was $7.9 \mathrm{mmol} / \mathrm{L}$ (2.5-7.8), creatinine concentration was $85 \mu \mathrm{mol} / \mathrm{L}$ (49-90), albumin concentration was $25 \mathrm{~g} / \mathrm{L}$ (3550) and C-reactive protein (CRP) concentration was 363 mg/L (normal <5). Her HIV status was negative. Her RAPID (R: renal factors [blood urea nitrogen]; A: age in years; P: purulence of pleural fluid; I: infection source [community or hospitalacquired]; D: dietary factors [serum albumin]) score was 4.

\section{TREATMENT}

The patient's azathioprineand hydroxychloroquine treatments were withheld and co-amoxiclav was prescribed, pending the analysis of the pus. A fortnightly review was planned. Her blood cultures were negative. The pleural fluid grew Streptococcus intermedius which was sensitive to amoxicillin and clindamycin. She did not have any further attempts at instrumentation of her chest and was treated for 6 weeks with oral amoxicillin and co-amoxiclav.
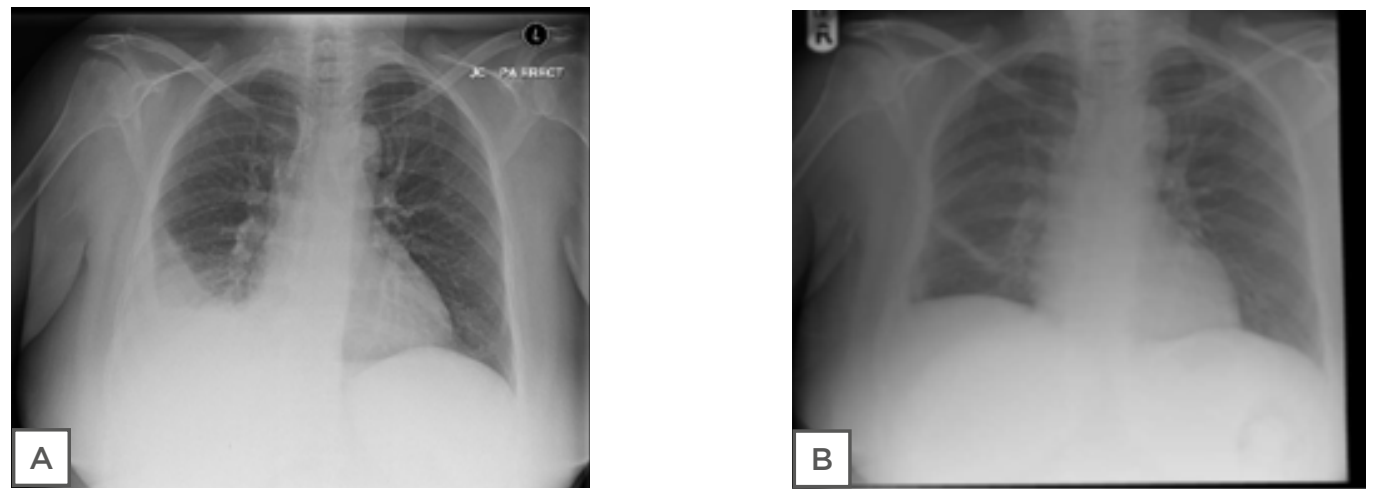

Figure 1: A) Chest radiograph showing large right sided pleural effusion. B) Chest radiography showing good resolution of the patient's effusion with minimal pleural reaction remaining.
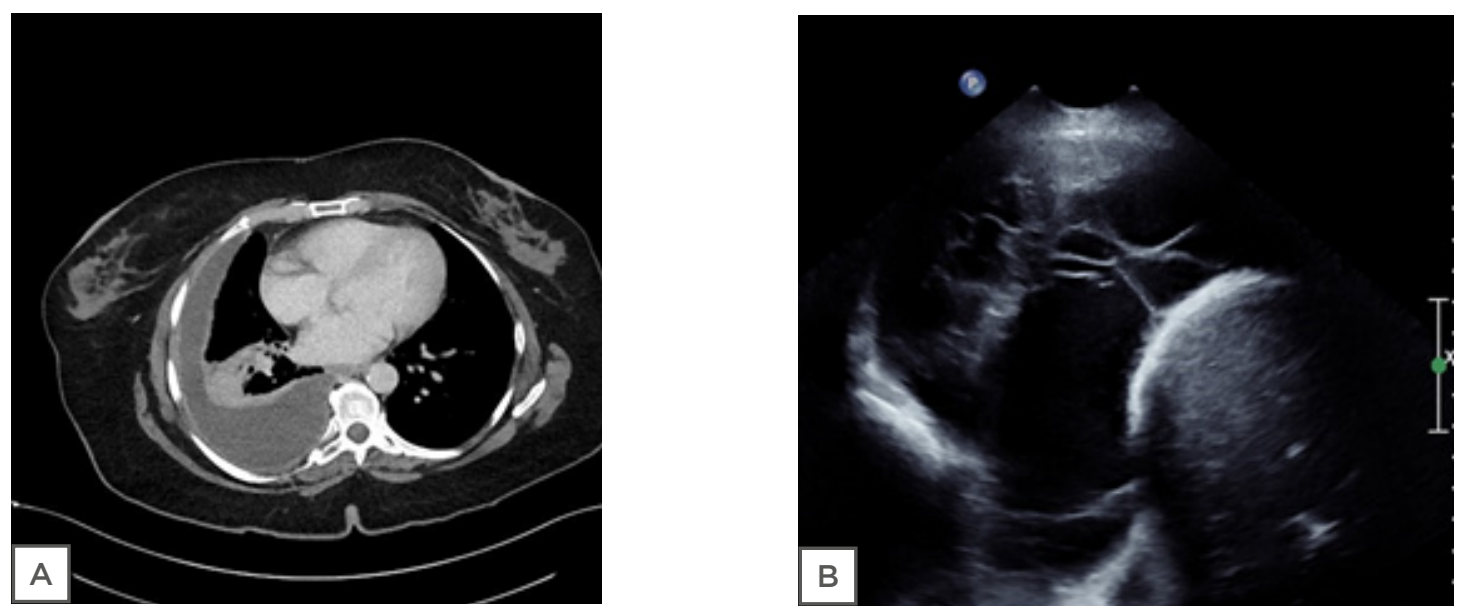

Figure 2: A) CT scan showing typical features of empyema with pleural enhancement and a lenticular effusion. B) Thoracic ultrasound showing loculations and hyperechoic effusion. 


\section{OUTCOME AND FOLLOW-UP}

Her white cell count and CRP improved upon antibiotic treatment, and she thus finished the course with no issues. Her final chest radiograph (Figure 1B) showed good resolution of her effusion with minimal pleural reaction remaining. Her white cell count and CRP normalised. Her immunosuppressants have been restarted and she continues under regular follow up.

\section{DISCUSSION}

Pleural infection or empyema is associated with significant morbidity and mortality and accrues long inpatient hospital stays. The 2010 British Thoracic Society (BTS) guidelines have provided a concise review of the literature available at the time. ${ }^{2}$

Management is dependent on drainage of any infected collection and provision of antibiotics, which should be tailored to any microbiological growth or towards the pathogens most likely to have caused the infection. As such, local microbiological and epidemiological data is important. 2,3

The authors audited local empyema cases to optimise local practice. Between December 2016 and December 2017, 36 patients were identified in the coding registry, with an average age of 64.5 years. A total of 19 patients were $>65$ years old. The most common past medical history included malignancy $(n=7)$ and alcohol excess $(n=5)$. Of the patients, 9 were current smokers and 16 were ex-smokers. No data was recorded for 5 of the patients. Recreational drug use was recorded for 1 patient. A total of 8 of the patients had an HIV test, all of whom were negative. HIV testing is strongly recommended in conditions associated with an undiagnosed HIV prevalence of greater than $0.1 \%$. Community acquired pneumonia and invasive pneumococcal disease form part of those conditions. ${ }^{4}$

No sampling of pleural fluid was done in six cases as the effusions were felt to be too small, and two attempts failed. Of the 28 samples available, fluid was frank pus or turbid in 13 cases, haemoserous in 5 , serous in 6 , and not commented on in 4 . $\mathrm{pH}$ results were available for 17 cases, and the $\mathrm{pH}$ was below 7.2 in 8 . Lactate dehydrogenase (LDH) levels were recorded in 14 samples.
BTS guidance recommends sampling of pleural fluid in all cases of pleural infection and that all samples should be sent for $\mathrm{pH}$, LDH, protein, glucose, and microbiology assessment. Pleural fluid analysis is crucial to the diagnosis, along with clinical and radiological features. Increased lactic acid formation, glucose, and anaerobic metabolism leads to a decrease in $\mathrm{pH}$, decrease in glucose, and an increase in LDH levels. ${ }^{2}$

Of the samples, 11 (39\%) were culture positive: 2 grew fully sensitive Streptococcus pneumonia, 1 grew S. intermedius, 1 previous intravenous drug user grew Actinomycosis turicensis and Haemophilus parainfluenzae, 1 grew Stenotrophomonas maltophilia as well as Klebsiella oxytoca, and 2 patients with indwelling pleural catheters tested positive for Staphylococcus aureus. Other organisms included S. intermedius, Streptococcus dysgalactiae, Streptococcus anginosus, and mixed anaerobes. All samples were sent in white topped bottles. Radiographic consolidation was noted in 23 patients. Two patients had indwelling pleural catheters.

It is important to know if any preceding parenchymal lung infection is community or hospital-acquired as the pathogens differ significantly. In a previous review, 1,523 patients with pleural infection and streptococcal infections were most commonly associated with community-acquired pneumonia, whereas $S$. aureus and Staphylococcus enterococcus were implicated in the hospital-acquired group. The median age of the patients was 64.0 years and the median percentage positivity of pleural fluid culture was $69 \%(48-77 \%)^{2,3}$

The authors' case agrees with this evidence, although our microbiological sensitivity is much lower than the quoted culture positivity. This is probably because samples were not concurrently sent in blood culture bottles. The addition of bottled blood culture to standard culture increases the proportion of patients with identifiable pathogens by $20.8 \%$. The authors have now incorporated this into their practice. ${ }^{5}$ Nine patients received antibiotics only. All patients received piperacillin/tazobactam or co-amoxiclav initially, except one who received tigecycline due to a penicillin allergy. All patients received $>2$ weeks of antibiotics, up to a maximum of 8 weeks. Clindamycin (combination 
or monotherapy) was prescribed in over half of all cases, but many organisms were gram negative and some of the Streptococcus strains were resistant. Clindamycin was also given for 6 weeks for fully sensitive Streptococcus strains. There is thus significant scope to tailor antibiotic prescriptions and adhere to local stewardship programmes. ${ }^{6}$ Local microbiological guidance suggests that, for sensitive strains, an addition of $500 \mathrm{mg}$ amoxicillin thrice daily to $625 \mathrm{mg}$ co-amoxiclav (amoxicillin/clavulanic acid) thrice daily achieves the oral equivalent dosage of 1.2 $\mathrm{g}$ intravenous co-amoxiclav. To provide excellent pleural penetration and be suitable for treatment of sensitive strains, $1.2 \mathrm{~g}$ is optimal. ${ }^{7}$

Drainage of any pleural effusion is recommended if the $\mathrm{pH}$ is less than 7.2 or if pus is present. This is based on expert opinion rather than objective, supportive evidence. ${ }^{2}$ In real-life clinical studies, up to $30 \%$ of such effusions are not drained due to technical difficulty or patient and physician choice. This is reflected in our practice. BTS guidance recommends the use of thoracic ultrasound for any intervention for pleural fluid. ${ }^{2}$ It is important to properly document any radiological findings. However, robust governance systems for thoracic ultrasound reporting do not exist; there is much intra- and inter-observer variation. Pleural fluid septation from pleural infection does not have an independent impact on mortality, although it may have an influence on other relevant clinical outcomes such as clearance of the pleural space and use of intra-pleural fibrinolytics. ${ }^{8}$ In the cohort, ultrasound findings were documented in only 15 notes. The most common comment was of multi-loculated fluid $(n=11)$. Other comments were 'small or moderate size' and 'echogenic.'

The mean length of stay was 9 weeks (range: 1-56 weeks). All survived to discharge after the first admission, 3 (9\%) had died within 30 days, and 3 more within 6 months. Eight patients were readmitted within 38 days: $75 \%$ due to ongoing pleural infection, with $50 \%$ of those staying for an average of 4 weeks. The total 6-month mortality of the cohort was $17 \%$. This is in line with known evidence. 9,10

A score for mortality prediction in pleural infection has recently been developed called the RAPID score." Patients with a RAPID score of 0-2 are considered low-risk, a score of 3-4 indicates a medium-risk, and a score of 5-7 indicates high-risk mortality at 3 months. Eight patients had a low-risk rapid score $(\leq 2)$, but no patients were managed as an outpatient. The score can be potentially used for risk stratification for outpatient management. As mentioned above, pus is thought to be an indication for immediate drainage, but recent reviews have argued that not all infected pleural spaces need to be drained and respond well to high dose antibiotics. ${ }^{10-12}$

\section{CONCLUSIONS}

To ensure compliance with various guidance available, we have produced a pleural procedure form (Figure 3) that encompasses all the recommendations from the above case review. ${ }^{12}$ Providing a safe and effective pleural service is a topical issue. Evison et al. $^{13}$ have provided a number of documents to ensure a pre-procedure checklist and adequate reporting of a pleural procedure. The authors believe this form is merely an adaptation of those and is better suited for local governance. ${ }^{14}$

The authors found significant room for improvement in their practice and believe that these findings can be applicable to any service dealing with pleural infection:

> Checking HIV status.

> Having a system for ultrasound reporting.

> Performing the required biochemical tests $(\mathrm{pH}$, LDH protein, glucose) on pleural fluid.

> Improving culture rates by sending fluid in blood bottles.

> Determing specific local mircrobiological epidemiology and applying correct antibiotic stewardship. 


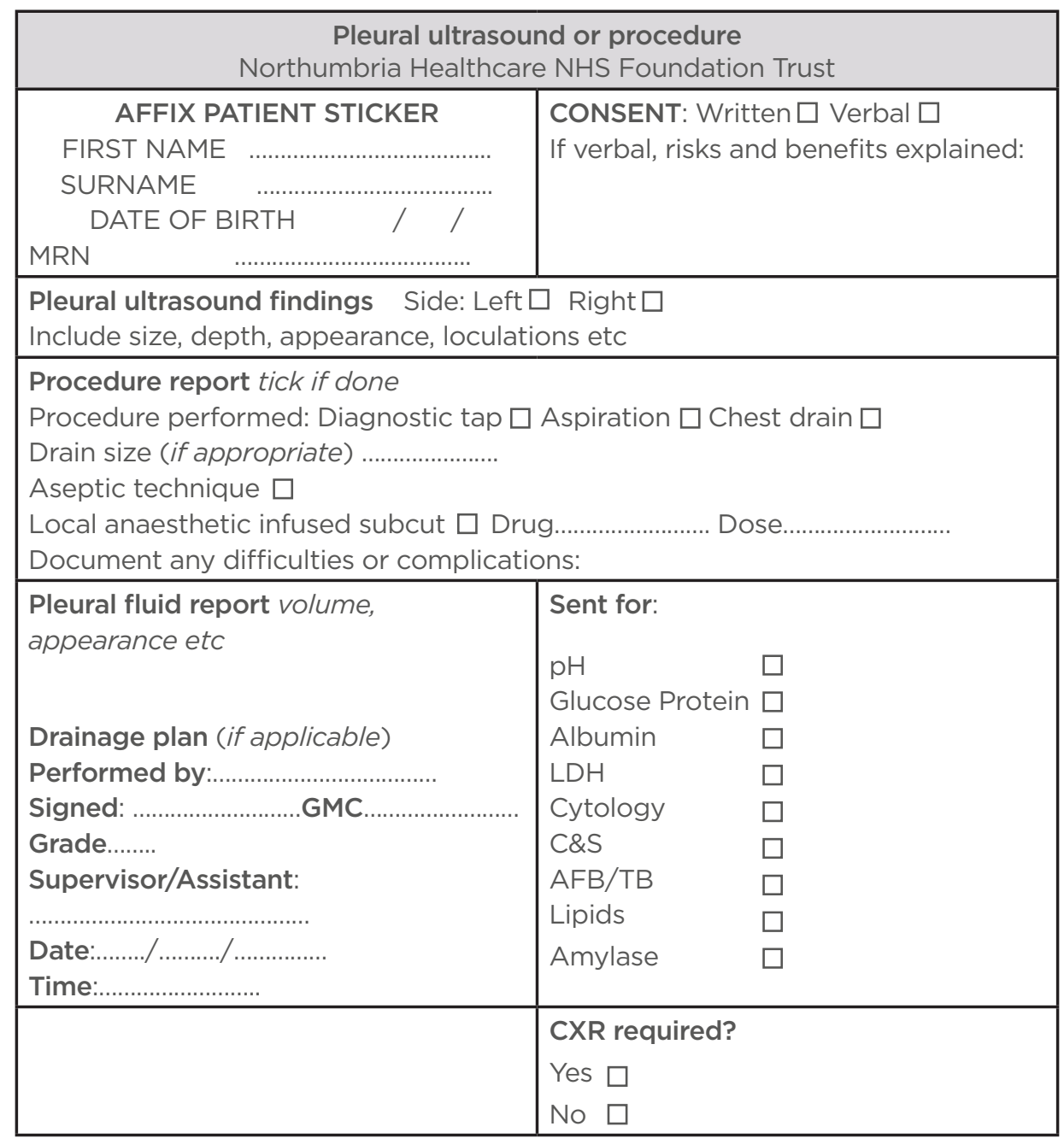

Figure 3: Northumbria Healthcare NHS Foundation Trust pleural procedure form.

\section{References}

1. Aujayeb $\mathrm{A}$ et al. A review of a pleural service. J R Coll Physicians Edinb. 2016;46(1):26-31

2. British Thoracic Society (BTS). BTS pleural disease guideline. Available at: https://www.brit-thoracic.org. uk/quality-improvement/guidelinearchive/. Last accessed: 16 May 2019.

3. Hassan M et al. Microbiology of pleural infection according to setting. Eur Respir J. 2018;58(suppl62):4713.

4. EurOTEST. HIV indicator conditions: Guidance for implementing HIV testing in adults in health care settings. Available at: http://www. eurotest.org/Portals/O/Indicator\%20 diseases/CHIP_Guidance\%20in\%20 short_UK_updated\%2OJUN2016_mlj. pdf. Last accessed: 26 March 2019.

5. Menzies SM et al. Blood culture bottle culture of pleural fluid in pleural infection. Thorax. 2011;66(8):658-62.

6. NHS: Health Education England. Combatting antimicrobial resistance: Educational approaches for the responsible prescribing of antimicrobials. Available at: https://www.hee.nhs.uk/ sites/default/files/documents/ Combatting\%2Oantimicrobial\%20 resistance $\% 20$ Educational\% 20 approaches $\% 2$ for $\% 20$ the $\% 20$ responsible\%20prescribing\%20of\%20 antimicrobials\%20-\%20full\%2Oreport. pdf. Last accessed: 26 March 2019.

7. Teixeira LR et al. Antibiotic levels in empyemic pleural fluid. Chest. 2000;117(6):1734-9.

8. Corcoran J et al. Does the sonographic presence and severity of pleural fluid septation have an impact on clinical outcomes in pleural infection? - Data from the Pleural Infection Longitudinal Outcome Study (PILOT). Eur Respir J. 2018;52supple62:OA3266

9. Bedawi EO et al. Recent developments in the management of pleural infection: A comprehensive review. Clin Respir J. 2018:12(8):230920

10. Ferreiro L et al. Management of pleural infections. Expert Rev Respir Med. 2018;12(6):521-35.

11. Rahman NM et al. A clinical score (RAPID) to identify those at risk for poor outcome at presentation in patients with pleural infection. Chest. 2014;145(4):848-55.

12. Fitzgerald DB, Lee YCG. Pleura infection: To drain or not to drain? 
Respirology. 2017;22(6):1055-6.

13. Evison $\mathrm{M}$ et al. Providing safe and effective pleural medicine services in the UK: An aspirational statement from UK pleural physicians. BMJ

Open Respir Res. 2018;5(1):e000307.

14. Aujayeb A. Pleural ultrasound or procedure reporting form. Available at: https://www.researchgate.net/ publication/332014027_Pleural_ ultrasound_or_procedure_reporting_ form. Last accessed: 7 March 2019. 